\title{
RIMAPS Analysis as a Tool to Elucidate Ascospore Ornamentation Pattern
}

\author{
S.M. Romero ${ }^{1,4}$, R.M. Comerio ${ }^{2}$ and E.A. Favret ${ }^{3,4}$ \\ ${ }^{1}$ Microbiología de Alimentos, Química Orgánica, FCEyN, UBA, Buenos Aires, Argentina \\ ${ }^{2}$ Instituto de Microbiología y Zoología Agrícola, CICVyA, INTA, Hurlingham, Argentina \\ ${ }^{3}$ Instituto de Suelos, CIRN, INTA. Hurlingham. Argentina \\ ${ }^{4}$ CONICET. Buenos Aires. Argentina
}

Fungal spores frequently present marked or sculptured surfaces; this feature is commonly referred as ornamentation [1]. The presence of surface bars, lines, spines, warts, etc. generally constitutes an important taxonomical diagnostic feature. On the other hand, the distribution pattern of ornamentation units on spore surface is critical as well. The focus of the present work has been placed on Neosartorya sp. ascospores ornamented with conical processes called spines (Fig. 1). The RIMAPS technique consists basically of rotating the image using algorithms of commercial software and calculating the $\mathrm{x}$-step of the two-dimensional Fourier transform for each $\mathrm{y}$-line of the new image obtained after rotation. As a consequence, averaged power spectra are obtained for each angular position. The corresponding maximum values are plotted as a function of rotation angle to obtain the RIMAPS spectrum. The maxima of the RIMAPS spectrum indicate main angular directions of the topographic pattern, the arrangement of the elements that constitute the surface [2]. RIMAPS has previously been used to recognize bars on ascospore surface [3]. In this opportunity, the objective was to apply RIMAPS to distinguish whether surface ascospore spines were distributed describing concentric rings or spirals, unrevealed patterns to the naked eye. The present work dealt with an original Neosartorya sp. spore image (Fig. 1), and with some conceptualized spore images as well. Mature cleistothecia of a Neosartorya sp. strain isolated from soil were crushed and coated with gold. The original image was obtained using a scanning electron microscope Zeiss Supra 40 with field emission gun. For spectra comparison purposes three handmade drawings were done: a copy of ornamentation edges (Fig. 2) that, as a heuristic tool, inspired the building up of two extra images with triangles and circles that served as testing models. In Fig. 3 triangles and circles follow a spiral pattern whereas in Fig. 4 they follow a concentric ring pattern. The number of elements of both images was exactly the same, besides the relative display of neighbouring elements was kept. RIMAPS spectra were taken from Figures 1, 3 and 4. Figure 5 shows the RIMAPS spectra of Figures 1 and 3. Many differences were observed between both spectra; particularly relevant were the MAPS values at $\left[75^{\circ}-105^{\circ}\right]$ and $\left[145^{\circ}-165^{\circ}\right]$. Figure 6 shows the RIMAPS spectra of Figures 1 and 4. In this case both spectra were almost coincident in all the angular range, meaning that the distribution of spines might be associated with a concentric ring pattern rather than a spiral one. In conclusion, RIMAPS analysis performs well as a tool to assign one of the two spore ornamentation patterns proposed. Nevertheless other extra patterns, such as double spirals, would be worth to analyze in order to effectively elucidate the arrangement of the spore-surface spines.

\section{References}

[1] PM Kirk et al., 2001, Ainsworth \& Bisby's Dictionary of the Fungi, (CAB International, Surrey, UK) 655 pp.

[2] NO Fuentes and EA Favret, Journal of Microscopy 206 (2002), p. 72-83.

[3] SM Romero et al., Proceedings of the $15^{\text {th }}$ European Microscopy Congress (September 2012). 


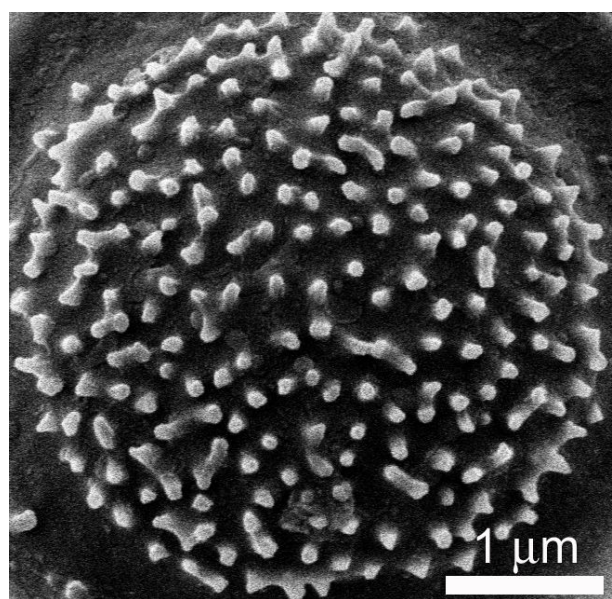

Figure 1. SEM micrograph of Neosartorya sp. ascospore

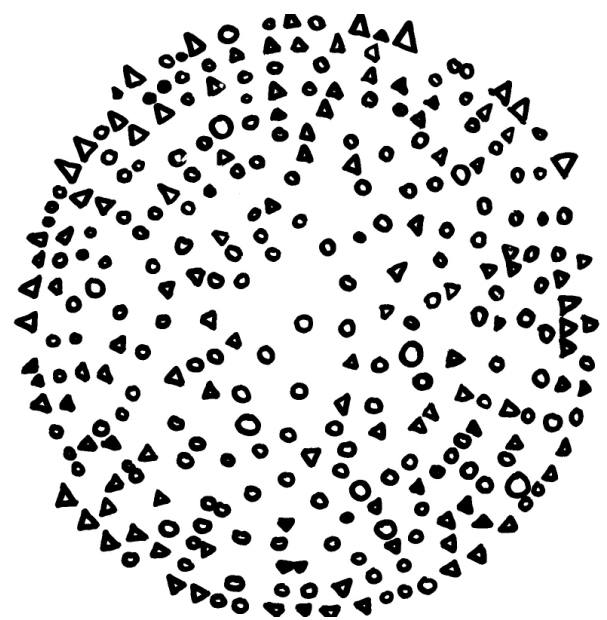

Figure 3. Testing model of the ascospore ornamentation following a spiral design

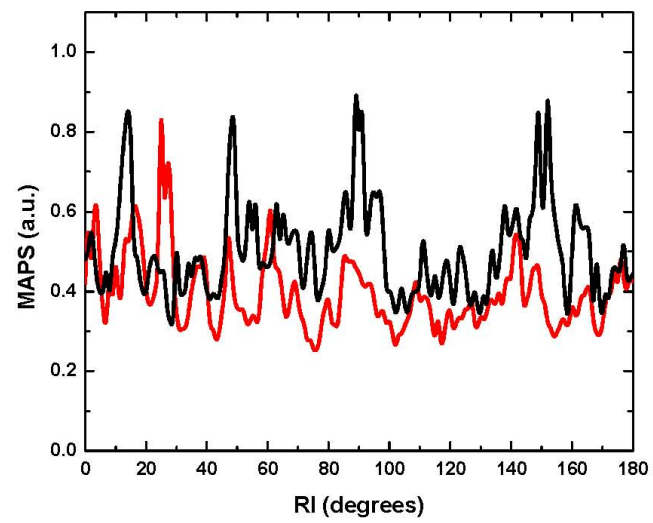

Figure 5. RIMAPS spectra of Figures 3 (black line) and 1 (red line)

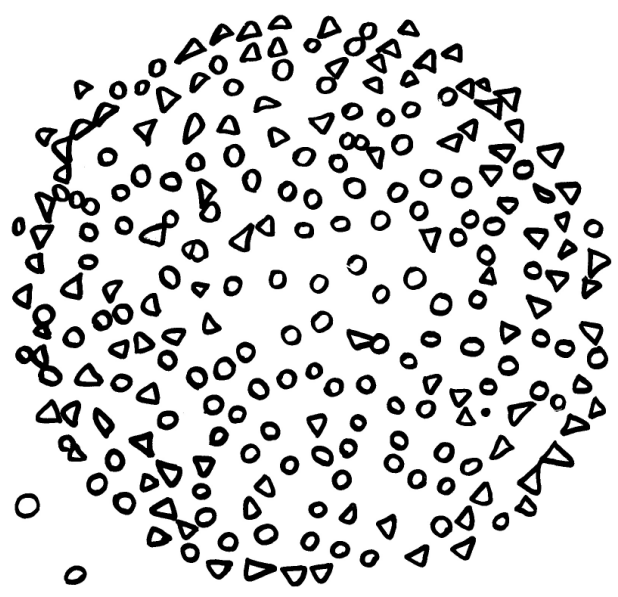

Figure 2. Copy of ornamentation edges of Figure 1

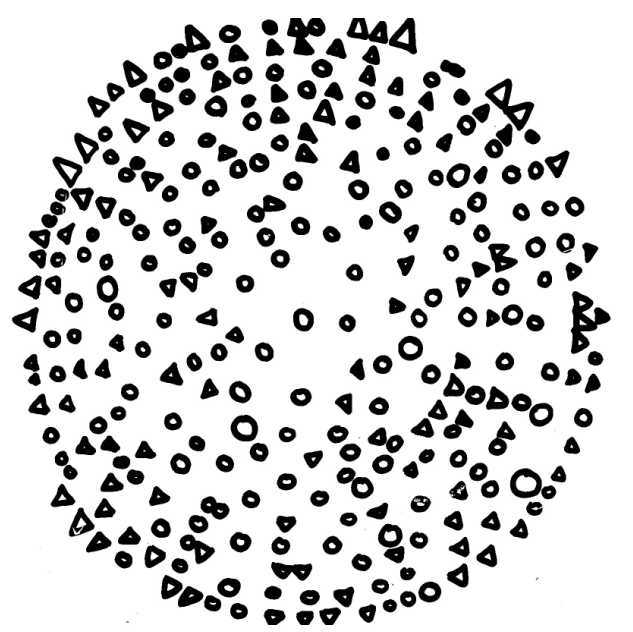

Figure 4. Testing model of the ascospore ornamentation following a concentric ring design

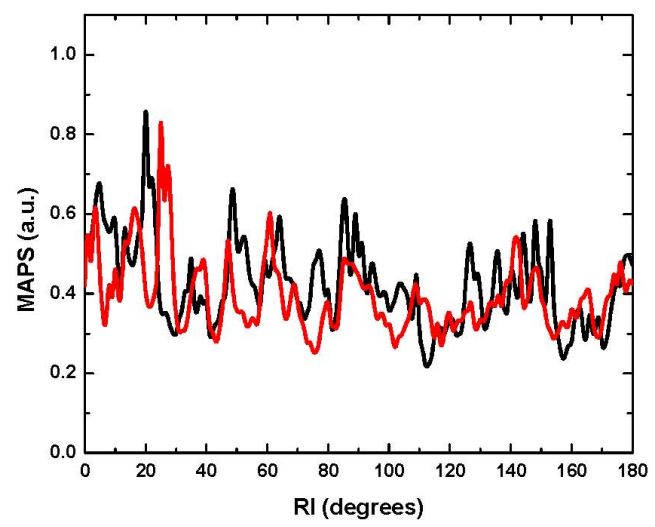

Figure 6. RIMAPS spectra of Figures 4 (black line) and 1 (red line) 